ISSN 1561-8323 (Print)

ISSN 2524-2431 (Online)

УДК 517.958

https://doi.org/10.29235/1561-8323-2019-63-2-142-149

Поступило в редакцию 18.02.2018

Received 18.02.2018

\author{
Академик В. И. Корзюк ${ }^{1}$ И. И. Столярчук ${ }^{2}$ \\ ${ }^{1}$ Институт математики Наџиональной академии наук Беларуси, Минск, Республика Беларусь \\ ${ }^{2}$ Белорусский государственный университет, Минск, Республика Беларусь \\ РЕШЕНИЕ СМЕШАННОЙ ЗАДАЧИ ДЛЯ УРАВНЕНИЯ ТИПА
КЛЕЙНА-ГОРДОНА-ФОКА С ИНТЕГРАЛЬНЫМИ УСЛОВИЯМИ
В СЛУЧАЕ НЕОДНОРОДНЫХ УСЛОВИЙ СОГЛАСОВАНИЯ
}

Аннотация. В данном сообщении рассматривается классическое решение смешанной задачи с интегральными условиями для уравнения типа Клейна-Гордона-Фока в полуполосе в случае, когда выполняются неоднородные условия согласования. Для рассматриваемой задачи строится эквивалентная задача сопряжения, в которой условия сопряжения задаются на характеристиках. Построенные неоднородные условия согласования однозначно определяют величину разрывов решения или его производных на характеристиках. Данные разрывы могут как сохраняться, так и сглаживаться с ростом аргумента по времени в зависимости от ядра интегрального оператора в нелокальных условиях. При решении указанной задачи возникают эквивалентные интегральные уравнения Вольтерры второго рода и их системы. Для полученных интегральных уравнений и систем существует единственное решение в классе дважды непрерывно дифференцируемых функций при заданной гладкости данных. При рассмотрении задачи использовался метод характеристик, который позволяет строить как точные, так и приближенные решения. Точные решения могут быть найдены в том случае, если удается разрешить эквивалентные интегральные уравнения Вольтерры. В противном случае можно найти приближенное решение задачи либо в аналитическом, либо в численном виде. При построении приближенного решения существенными оказываются условия согласования, которые необходимо учитывать при использовании численных методов решения задачи.

Ключевые слова: уравнение Клейна-Гордона-Фока, метод характеристик, условия сопряжения, классическое решение, смешанная задача, условия согласования

Для цитирования. Корзюк, В. И. Решение смешанной задачи для уравнения типа Клейна-Гордона-Фока с интегральными условиями в случае неоднородных условий согласования / В. И. Корзюк, И. И. Столярчук // Докл. Нац. акад. наук Беларуси. - 2019. - Т. 63, № 2. - С. 142-149. https://doi.org/10.29235/1561-8323-2019-63-2-142-149

Academician Viktor I. Korzyuk ${ }^{1}$, Ivan I. Stolyarchuk ${ }^{2}$

${ }^{I}$ Institute of Mathematics of the National Academy of Sciences of Belarus, Minsk, Republic of Belarus ${ }^{2}$ Belarusian State University, Minsk, Republic of Belarus

\title{
SOLVING THE MIXED PROBLEM FOR THE KLEIN-GORDON-FOCK TYPE EQUATION WITH INTEGRAL CONDITIONS IN THE CASE OF THE INHOMOGENEOUS MATCHING CONDITIONS
}

Abstract. The classical solution of the mixed problem with integral conditions for the Klein-Gordon-Fock type equation in the half strip is considered when inhomogeneous matching conditions are fulfilled. An equivalent conjugation problem is formulated where conjugation conditions are set on characteristics. Constructed inhomogeneous conditions uniquely define gaps of the solution or its derivatives on characteristics and given gaps can be either remained or smoothed while the time argument increases depending on the kernel of the integral operator in unlocal conditions. The solution of this problem is reduced to solving the second-type Volterra integral equations and their systems. The unique solution of these equations in the class of the twice continuously differentiable functions exists when the initial functions are smooth enough. While considering the given problem the method of characteristics is used to construct both an analytical solution, when the solution of the integral equation can be found explicitly, and an approximate solution. Moreover, approximate solutions can be constructed in numerical and analytical form. When the numerical solution is constructed, matching conditions are significant and need to be considered while developing numerical methods.

Keywords: Klein-Gordon-Fock equation, characteristics method, conjugation conditions, classical solution, mixed problem, matching conditions

For citation: Korzyuk V. I., Stolyarchuk I. I. Solving the mixed problem for the Klein-Gordon-Fock type equation with integral conditions in the case of the inhomogeneous matching conditions. Doklady Natsional'noi akademii nauk Belarusi = Doklady of the National Academy of Sciences of Belarus, 2019, vol. 63, no. 2, pp. 142-149 (in Russian). https://doi.org/10.29235/ 1561-8323-2019-63-2-142-149

(С Корзюк В. И., Столярчук И. И., 2019 
Введение. В [1-5] с помощью метода характеристик исследуются различные смешанные задачи для уравнения Клейна-Гордона-Фока. Для каждой из рассматриваемых задач получены условия согласования, которые при достаточной гладкости заданных функций являются необходимыми и достаточными условиями для принадлежности решения классу дважды непрерывно дифференцируемых функций.

В [6, с. 13-20] рассмотрен случай неоднородных условий согласования для первой смешанной задачи волнового уравнения. В результате была поставлена корректная задача сопряжения, доказано существование и единственность классического решения первой смешанной задачи в случае, когда выполняются неоднородные условия согласования.

В данном сообщении метод характеристик применяется для изучения неоднородных условий согласования смешанной задачи для уравнения Клейна-Гордона-Фока с нелокальными условиями.

Постановка задачи. Задача рассматривается на плоскости двух независимых переменных $t, x$. На замыкании $\bar{Q}$ области $Q=(0 ; l) \times \mathbb{R}^{+}, l \in \mathbb{R}, l<+\infty$, задается одномерное уравнение типа Клейна-Гордона-Фока

$$
L v=L^{(0)} v-\lambda(t, x) v=\partial_{t}^{2} v-a^{2} \partial_{x}^{2} v-\lambda(t, x) v=f(t, x),
$$

где $\lambda$ и $f$ - функции, заданные на множестве $\bar{Q}=[0 ; \infty) \times[0 ; l] \subset \mathbb{R}^{2}=\mathbb{R} \times \mathbb{R} ; \mathbb{R}-$ множество действительных чисел.

С помощю прямых $t=k l / a, k=0,1, \ldots$, область $Q$ разбивается на подобласти $Q^{(k)}$. Далее с помощью характеристик $x-a t=-k l, x+a t=(k+1) l, k=0,1, \ldots$, каждая из подобластей $Q^{(k)}$ разбивается на непересекающиеся подмножества $Q^{(k, j)}$, такие, что $\bar{Q}=\bigcup_{k=0}^{\infty} \bigcup_{j=1}^{4} \overline{Q^{(k, j)}}$.

К уравнению (1) присоединяются условия Коши

$$
v(0, x)=\varphi(x), \partial_{t} v(0, x)=\psi(x), \quad x \in[0 ; l],
$$

и нелокальные условия

$$
v(t, j)=\int_{0}^{l} K_{j}(t, s) v(t, s) d s+\widetilde{q^{(j)}}(t), \quad t \in[0 ; \infty), j \in\{0, l\},
$$

где $K_{j}(t, x) \widetilde{q^{(j)}}(t), j \in\{0, l\}, \varphi(x), \psi(x)$ - некоторые наперед заданные функции, гладкость которых будет уточнена ниже.

Как было показано в [4; 5], задача (1)-(3) сводится к решению задачи для однородного уравнения

$$
\begin{gathered}
\partial_{t}^{2} u-a^{2} \partial_{x}^{2} u-\lambda(t, x) u=0, \\
u(0, x)=\varphi(x), \partial_{t} u(0, x)=\psi(x), \quad x \in[0 ; l], \\
u(t, j)=\int_{0}^{l} K_{j}(t, s) u(t, s) d s+q^{(j)}(t), \quad t \in[0 ; \infty), j \in\{0, l\},
\end{gathered}
$$

где $q^{(j)}(t)=\widetilde{q^{(j)}}(t)-\int_{0}^{l} K_{j}(t, s) w(t, s) d s-w(t, j), j \in\{0, l\}$. Здесь $w(t, j)-$ решение следующей задачи для неоднородного уравнения

$$
\partial_{t}^{2} w-a^{2} \partial_{x}^{2} w-\lambda(t, x) w=f(t, x)
$$

с однородными начальными условиями

$$
w(0, x)=0, \partial_{t} w(0, x)=0, \quad x \in[0 ; l] .
$$

Доказательство существования дважды непрерывно дифференцируемого решения задачи (7)-(8) приведено в [5]. 
В $[4 ; 5]$ была доказана теорема

Т е о р е м а 1 . Пусть $\lambda \in C^{1}(\bar{Q}), q^{(j)} \in C^{2}([0 ;+\infty)), K_{j} \in C^{2}(\bar{Q}), j \in\{0, l\}, \quad \varphi \in C^{2}([0 ; l])$, $\psi \in C^{1}([0 ; l])$. Решение задачи (4)-(6) существует и единственно в классе $C^{2}(\bar{Q})$ тогда и только тогда, когда выполняются однородные условия согласования

$$
\begin{gathered}
q^{(0)}(0)-\varphi(0)+\int_{0}^{l} K_{0}(0, s) \varphi(s) d s=0, q^{(l)}(0)-\varphi(l)+\int_{0}^{l} K_{l}(0, s) \varphi(s) d s=0, \\
-\frac{1}{a} d q^{(0)}(0)+\frac{1}{a} \psi(0)-\frac{1}{a} \int_{0}^{l}\left(\partial_{t} K_{0}(0, s) \varphi(s)+\psi(s) K_{0}(0, s)\right) d s=0 \\
\frac{1}{a} d q^{(l)}(0)-\frac{1}{a} \psi(l)-\frac{1}{a} \int_{0}^{l}\left(\partial_{t} K_{l}(0, s) \varphi(s)+\psi(s) K_{l}(0, s)\right) d s=0 \\
\frac{1}{a^{2}} d^{2} q^{(0)}(0)-d^{2} \varphi(0)-\frac{1}{a^{2}} \varphi(0) \lambda(0,0)+ \\
+\frac{1}{a^{2}} \int_{0}^{l}\left(\partial_{t}^{2} K_{0}(0, s) \varphi(s)+2 \partial_{t} K_{0}(0, s) \psi(s)+K_{0}(0, s)\left(a^{2} d^{2} \varphi(s)+\varphi(s) \lambda(0, s)\right)\right) d s=0 \\
\frac{1}{a^{2}} d^{2} q^{(l)}(0)-d^{2} \varphi(l)-\frac{1}{a^{2}} \varphi(l) \lambda(0, l)+ \\
+\frac{1}{a^{2}} \int_{0}^{l}\left(\partial_{t}^{2} K_{l}(0, s) \varphi(s)+2 \partial_{t} K_{l}(0, s) \psi(s)+K_{l}(0, s)\left(a^{2} d^{2} \varphi(s)+\varphi(s) \lambda(0, s)\right)\right) d s=0
\end{gathered}
$$

При этом решение $u(t, x)$ задачи (4)-(6) определяется как $u(t, x)=u^{(k)}(t, x),(t, x) \in \overline{Q^{(k)}}$, где $u^{(k)}(t, x)$ задается формулой

$$
u^{(k)}(t, x)=-\frac{1}{4 a^{2}} \int_{-k l}^{x-a t} \int_{(k+1) l}^{x+a t}\left(\lambda u^{(k)}\right)\left(\frac{\eta-\xi}{2 a}, \frac{\eta+\xi}{2}\right) d \eta d \xi+p^{(k)}(x-a t)+g^{(k)}(x+a t)
$$

при соответствующем выборе значений функций $p^{(k)}, g^{(k)}, k=0,1, \ldots$.

Неоднородные условия согласования. Рассмотрим неоднородные условия согласования в области $Q^{(k)}$

$$
\begin{gathered}
q^{(0)}\left(\frac{k l}{a}\right)-\varphi^{(k)}(0)+\int_{0}^{l} K_{0}\left(\frac{k l}{a}, s\right) \varphi^{(k)}(s) d s=\sigma_{0}^{(k)}, q^{(l)}\left(\frac{k l}{a}\right)-\varphi^{(k)}(l)+\int_{0}^{l} K_{l}\left(\frac{k l}{a}, s\right) \varphi^{(k)}(s) d s=\delta_{0}^{(k)}, \\
-\frac{1}{a} d q^{(0)}\left(\frac{k l}{a}\right)+\frac{1}{a} \psi^{(k)}(0)-\frac{1}{a} \int_{0}^{l}\left(\partial_{t} K_{0}\left(\frac{k l}{a}, s\right) \varphi^{(k)}(s)+\psi^{(k)}(s) K_{0}\left(\frac{k l}{a}, s\right)\right) d s=\sigma_{1}^{(k)}, \\
\frac{1}{a} d q^{(l)}\left(\frac{k l}{a}\right)-\frac{1}{a} \psi^{(k)}(l)-\frac{1}{a} \int_{0}^{l}\left(\partial_{t} K_{l}\left(\frac{k l}{a}, s\right) \varphi^{(k)}(s)+\psi^{(k)}(s) K_{l}\left(\frac{k l}{a}, s\right)\right) d s=\delta_{1}^{(k)}, \\
\frac{1}{a^{2}} d^{2} q^{(0)}\left(\frac{k l}{a}\right)-d^{2} \varphi^{(k)}(0)-\frac{1}{a^{2}} \varphi^{(k)}(0) \lambda\left(\frac{k l}{a}, 0\right)+ \\
+\frac{1}{a^{2}} \int_{0}^{l}\left(\partial_{t}^{2} K_{0}\left(\frac{k l}{a}, s\right) \varphi^{(k)}(s)+2 \partial_{t} K_{0}\left(\frac{k l}{a}, s\right) \psi(k)(s)+K_{0}\left(\frac{k l}{a}, s\right) \times\right. \\
\left.\times\left(a^{2} d^{2} \varphi^{(k)}(s)+\varphi^{(k)}(s) \lambda\left(\frac{k l}{a}, s\right)\right)\right) d s=\sigma_{2}^{(k)}, \\
\frac{1}{a^{2}} d^{2} q^{(l)}\left(\frac{k l}{a}\right)-d^{2} \varphi^{(k)}(l)-\frac{1}{a^{2}} \varphi^{(k)}(l) \lambda\left(\frac{k l}{a}, l\right)+
\end{gathered}
$$




$$
\begin{aligned}
+\frac{1}{a^{2}} \int_{0}^{l}\left(\partial_{t}^{2}\right. & K_{l}\left(\frac{k l}{a}, s\right) \varphi^{(k)}(s)+2 \partial_{t} K_{l}\left(\frac{k l}{a}, s\right) \psi^{(k)}(s)+K_{l}\left(\frac{k l}{a}, s\right) \times \\
\times & \left.\left(a^{2} d^{2} \varphi^{(k)}(s)+\varphi^{(k)}(s) \lambda\left(\frac{k l}{a}, s\right)\right)\right) d s=\delta_{2}^{(k)}
\end{aligned}
$$

где $\varphi^{(k)}(x)=u^{(k-1)}\left(\frac{k l}{a}, x\right), \psi^{(k)}(x)=\partial_{t} u^{(k-1)}\left(\frac{k l}{a}, x\right), \sigma_{i}^{(k)}, \delta_{i}^{(k)} \in \mathbb{R}, i=\overline{0,2}, k=1,2, \ldots$.

Л е м м а 1. Пусть в области $Q^{(k)}$ справедливы неоднородные условия согласования (10) и выполняются условия на гладкость функичй $\lambda \in C^{1}(\bar{Q}), q^{(j)} \in C^{2}([0 ;+\infty)), K_{j} \in C^{2}(\bar{Q}), j \in\{0, l\}$, $\varphi^{(k)} \in C^{2}([0 ; l]), \psi^{(k)} \in C^{1}([0 ; l])$. Тогда для решения $u^{(k, j)}(t, x),(t, x) \in Q^{(k, j)}$, задачи (4)-(6) справедливы следуюшие условия сопряжения:

$$
\begin{aligned}
& u^{(k, j+1)}(t, a t-k l)-u^{(k, j)}(t, a t-k l)=\sigma_{0}^{(k)}, \\
& \partial_{x} u^{(k, j+1)}(t, a t-k l)-\partial_{x} u^{(k, j)}(t, a t-k l)=\sigma_{1}^{(k)}-\frac{\sigma_{0}^{(k)}}{4 a^{2}} \int_{k l}^{-k l+2 a t} \lambda\left(\frac{\eta+k l}{2 a}, \frac{\eta-k l}{2}\right) d \eta, \\
& \partial_{x}^{2} u^{(k, j+1)}(t, a t-k l)-\partial_{x}^{2} u^{(k, j)}(t, a t-k l)=\sigma_{2}^{(k)}+\frac{\sigma_{1}^{(k)}}{4 a^{2}} \int_{-k l+2 a t}^{k l} \lambda\left(\frac{\eta+k l}{2 a}, \frac{\eta-k l}{2}\right) d \eta+ \\
& +\frac{\sigma_{0}^{(k)}}{2 a^{2}}\left(\frac { 1 } { 4 } \int _ { - k l + 2 a t } ^ { k l } \left(\partial_{x} \lambda\left(\frac{\eta+k l}{2 a}, \frac{\eta-k l}{2}\right)-\frac{1}{a} \partial_{t} \lambda\left(\frac{\eta+k l}{2 a}, \frac{\eta-k l}{2}\right)-\right.\right. \\
& \left.\left.-\frac{1}{2 a^{2}} \int_{k l}^{2 a \eta^{-k l}} \lambda\left(\frac{\eta_{1}+k l}{2 a}, \frac{\eta_{1}-k l}{2}\right) d \eta_{1} \times \lambda\left(\frac{\eta+k l}{2 a}, \frac{\eta-k l}{2}\right)\right) d \eta+\lambda\left(\frac{k l}{a}, 0\right)-\lambda(t,-k l+a t)\right), \\
& j \in\{1,3\}, t \in\left[\frac{k l}{a} ; \frac{(k+1) l}{a}\right], \\
& u^{(k, j+2)}(t,(k+1) l-a t)-u^{(k, j)}(t,(k+1) l-a t)=\delta_{0}^{(k)}, \\
& \partial_{x} u^{(k, j+2)}(t,(k+1) l-a t)-\partial_{x} u^{(k, j)}(t,(k+1) l-a t)=\delta_{1}^{(k)}+\frac{\delta_{0}^{(k)}}{4 a^{2}} \int_{(k+1) l-2 a t}^{-(k-1) l} \lambda\left(\frac{(k+1) l-\xi}{2 a}, \frac{(k+1) l+\xi}{2}\right) d \xi \\
& \partial_{x}^{2} u^{(k, j+2)}(t,(k+1) l-a t)-\partial_{x}^{2} u^{(k, j)}(t,(k+1) l-a t)=\delta_{2}^{(k)}+\frac{\delta_{1}^{(k)}}{4 a^{2}} \int_{(k+1) l-2 a t}^{-(k-1) l} \lambda\left(\frac{(k+1) l-\xi}{2 a}, \frac{(k+1) l+\xi}{2}\right) d \xi+ \\
& +\frac{\delta_{0}^{(k)}}{2 a^{2}}\left(\int _ { ( k + 1 ) l - 2 a t } ^ { - ( k - 1 ) l } \frac { 1 } { 4 } \left(\partial_{x} \lambda\left(\frac{(k+1) l-\xi}{2 a}, \frac{(k+1) l+\xi}{2}\right)+\frac{1}{a} \partial_{t} \lambda\left(\frac{(k+1) l-\xi}{2 a}, \frac{(k+1) l+\xi}{2}\right)+\right.\right. \\
& \left.+\frac{1}{2 a^{2}} \lambda\left(\frac{(k+1) l-\xi}{2 a}, \frac{(k+1) l+\xi}{2}\right) \int_{(k+1) l-2 a \xi}^{-(k-1) l} \lambda\left(\frac{(k+1) l-\xi_{1}}{2 a}, \frac{(k+1) l+\xi_{1}}{2}\right) d \xi_{1}\right) d \xi+ \\
& \left.+\lambda\left(\frac{k l}{a}, l\right)-\lambda(t,(k+1) l-a t)\right), j \in\{1,2\}, t \in\left[\frac{k l}{a} ; \frac{(k+1) l}{a}\right],
\end{aligned}
$$

где константы $\delta_{j}^{(k)}, \sigma_{j}^{(k)}, j=\overline{0,2}$, определяются по (10).

С л е д с т в и е 1. Какими бы ни были гладкими функиии $q^{(j)}(t), \varphi^{(k)}(x), \psi^{(k)}(x), K_{j}, j \in\{0, l\}$, в задаче (4)-(6) при выполнении неравенства $\sum_{j=0}^{2}\left(\sigma_{j}^{(k)}\right)^{2}+\left(\delta_{j}^{(k)}\right)^{2} \neq 0$, не существует классического решения задачи (4)-(6), определенного на $\overline{Q^{(k)}}$. 
Рассмотрим следующую область: $\tilde{Q}=\{(t, x) \in Q \mid x+a t \neq(k+1) l \wedge x-a t \neq-k l, k=0,1,2, \ldots\}$. Для дальнейших рассуждений понадобятся множества $\widetilde{Q^{(k)}}=\overline{Q^{(k)}} \cap \tilde{Q}$.

С л е д с т в и е 2. Пусть в области $Q^{(k)}$ функциии $\lambda \in C^{1}(\bar{Q}), q^{(j)} \in C^{2}([0 ;+\infty))$,

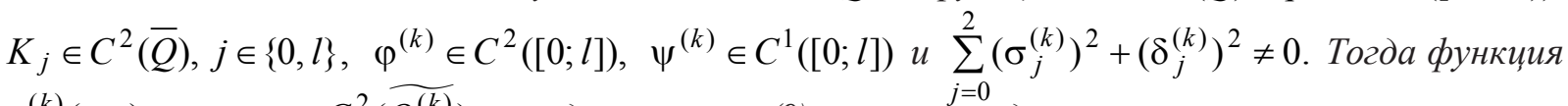
$u^{(k)}(t, x)$ из класса $C^{2}\left(\widetilde{Q^{(k)}}\right)$, определенная по (9), является единственным классическим решением задачи (4)-(6) на $\widehat{Q^{(k)}}$ тогда и только тогда, когда выполнены условия согласования (10).

3 а м е ч а н и е. Если $\sigma_{0}^{(k)}=\delta_{0}^{(k)}=0 u \sum_{j=1}^{2}\left(\sigma_{j}^{(k)}\right)^{2}+\left(\delta_{j}^{(k)}\right)^{2} \neq 0$, то функция $u^{(k)}(t, x)$ из класса $C(\bar{Q}) \bigcap C^{2}\left(\widetilde{Q^{(k)}}\right)$, определенная по (9), является единственным классическим решением задачи (4)-(6) на $\widehat{Q^{(k)}}$ тогда и только тогда, когда выполнены условия согласования (10).

Можно сформулировать аналогичные замечания для различных случаев, когда какие-либо из значений $\sigma_{j}^{(k)}$ и $\delta_{j}^{(k)}$ обращаются в нуль.

Далее показана зависимость скачков $\sigma_{j}^{(k)}, \delta_{j}^{(k)}$ от $\sigma_{j}^{(k-1)}, \delta_{j}^{(k-1)}, j=\overline{0,2}$.

Л е м м а 2. Пусть в области $Q^{(k-1)}$ выполняются неоднородные условия согласования (10). Тогда справедливы следующие выражения, определяющие зависимость $\sigma_{j}^{(k)}$ от $\delta_{j}^{(k-1)}, j=\overline{0,2}$ :

$$
\begin{gathered}
\sigma_{0}^{(k)}=-\delta_{0}^{(k-1)}\left(1+\int_{0}^{l} K_{l}\left(\frac{(k-1) l}{a}, s\right) d s\right) \\
\sigma_{1}^{(k)}=\delta_{1}^{(k-1)}\left(1+\int_{0}^{l} K_{l}\left(\frac{(k-1) l}{a}, s\right) d s\right)+\frac{\delta_{0}^{(k-1)}}{4 a^{2}} \int_{-k l}^{2 l-k l} \lambda\left(\frac{k l-\xi}{2 a}, \frac{k l+\xi}{2}\right) d \xi+\frac{\delta_{0}^{(k-1)} l}{a} \int_{0}^{l} \partial_{t} K_{l}\left(\frac{(k-1) l}{a}, s\right) d s, \\
\sigma_{2}^{(k)}=-\delta_{2}^{(k-1)}\left(1+\int_{0}^{l} K_{l}\left(\frac{(k-1) l}{a}, s\right) d s\right)+\frac{1}{16 a^{4}} \int_{2 l-k l}^{-k l}\left(4 a^{2} \delta_{1}^{(k-1)}+\delta_{0}^{(k-1)} \int_{\xi}^{2 l-k l} \lambda\left(\frac{k l-\tau}{2 a}, \frac{k l+\tau}{2}\right) d \tau\right) \times \\
\times \lambda\left(\frac{k l-\xi}{2 a}, \frac{k l+\xi}{2}\right) d \xi+\frac{\delta_{0}^{(k-1)}}{8 a^{2}} \int_{2 l-k l}^{-k l} \partial_{x} \lambda\left(\frac{k l-\xi}{2 a}, \frac{k l+\xi}{2}\right)+\frac{1}{a} \partial_{t} \lambda\left(\frac{k l-\xi}{2 a}, \frac{k l+\xi}{2}\right) d \xi+ \\
\quad+\frac{\delta_{0}^{(k-1)}}{2 a^{2}}\left(\lambda\left(\frac{k l}{a}, 0\right)+\lambda\left(\frac{(k-1) l}{a}, l\right)\right)-\frac{\delta_{0}^{(k-1)}}{2 a^{2}} \lambda\left(\frac{k l}{a}, 0\right)_{0}^{l} \int_{l} K_{l}\left(\frac{(k-1) l}{a}, s\right) d s- \\
-\frac{1}{a^{2}} \int_{0}^{l} \partial_{t}^{2} K_{l}\left(\frac{k l}{a}, s\right) \delta_{0}^{(k-1)}+2 \partial_{t} K_{l}\left(\frac{(k-1) l}{a}, s\right) \delta_{1}^{(k-1)}+K_{l}\left(\frac{(k-1) l}{a}, s\right) \delta_{0}^{(k-1)} \lambda\left(\frac{(k-1) l}{a}, s\right) d s,
\end{gathered}
$$

а также зависимость $\delta_{j}^{(k)}$ oт $\sigma_{j}^{(k-1)}, j=\overline{0,2}$ :

$$
\begin{gathered}
\delta_{0}^{(k)}=-\sigma_{0}^{(k-1)}\left(1+\int_{0}^{l} K_{0}\left(\frac{(k-1) l}{a}, s\right) d s\right) \\
\delta_{1}^{(k)}=-\sigma_{1}^{(k-1)}\left(1+\int_{0}^{l} K_{0}\left(\frac{(k-1) l}{a}, s\right) d s\right)+\frac{\sigma_{0}^{(k-1)}}{4 a^{2}} \int_{(k+1) l}^{(k-1) l} \lambda\left(\frac{\eta+(k-1) l}{2 a}, \frac{\eta-(k-1) l}{2}\right) d \eta- \\
-\frac{\sigma_{0}^{(k-1)} l}{a} \int_{0}^{l} \partial_{t} K_{0}\left(\frac{(k-1) l}{a}, s\right) d s \\
\delta_{2}^{(k)}=-\sigma_{2}^{(k-1)}\left(1+\int_{0}^{l} K_{0}\left(\frac{(k-1) l}{a}, s\right) d s\right)+\frac{\sigma_{0}^{(k-1)}}{2 a^{2}}\left(\lambda\left(\frac{k l}{a}, l\right)+\lambda\left(\frac{(k-1) l}{a}, 0\right)\right)+
\end{gathered}
$$




$$
\begin{gathered}
+\frac{\sigma_{0}^{(k-1)}}{8 a^{2}} \int_{(k+1) l}^{(k-1) l} \partial_{x} \lambda\left(\frac{\eta+(k-1) l}{2 a}, \frac{\eta-(k-1) l}{2}\right)+\frac{1}{a} \partial_{t} \lambda\left(\frac{\eta+(k-1) l}{2 a}, \frac{\eta-(k-1) l}{2}\right) d \eta+ \\
+\frac{1}{16 a^{4}} \int_{(k+1) l}^{(k-1) l} \lambda\left(\frac{\eta+(k-1) l}{2 a}, \frac{\eta-(k-1) l}{2}\right)\left(-4 a^{2} \sigma_{1}^{(k-1)}+\sigma_{0}^{(k-1)} \int_{(k-1) l}^{\eta} \lambda\left(\frac{\tau+(k-1) l}{2 a}, \frac{\tau-(k-1) l}{2}\right) d \tau\right) d \eta+ \\
+\frac{1}{a^{2}} \int_{0}^{l} \partial_{t}^{2} K_{0}\left(\frac{(k-1) l}{a}, s\right) \sigma_{0}^{(k-1)}+2 \partial_{t} K_{0}\left(\frac{(k-1) l}{a}, s\right) \sigma_{1}^{(k-1)}+K_{0}\left(\frac{(k-1) l}{a}, s\right) \times \\
\times\left(\sigma_{0}^{(k-1)} \lambda\left(\frac{(k-1) l}{a}, s\right)\right) d s+\frac{\sigma_{0}^{(k-1)}}{2 a^{2}} \lambda\left(\frac{(k-1) l}{a}, l\right) \int_{0}^{l} K_{0}\left(\frac{(k-1) l}{a}, s\right) d s .
\end{gathered}
$$

С л е д с т в и е 3. Условия согласования (10) выполняются при некотором $k$, тогда и только тогда, когда они выполняются для $k-1$.

С л е д с т в и е 4. Если для некоторого $k$ выполняются однородные условия согласования, то для всех $m>k, m \in \mathbb{N}$, выполняются однородные условия согласования.

С л е д с т в и е 5. Если существуют такие номера $k_{j}^{(i)}, i \in\{0, l\}, j=\overline{1,3}$, причем $k_{j_{1}}^{(i)} \neq k_{j_{2}}^{(i)}, j_{1} \neq j_{2}$, что $\int_{0}^{l} K_{i}\left(\frac{\left(k_{j}^{(i)}-1\right) l}{a}, s\right) d s=-1, \operatorname{mo} u(t, x) \in C^{2}\left(\bigcup_{k=\max \left(k_{j}^{(i)}\right)}^{\infty} \overline{Q^{(k)}}\right), i=0, l$.

С л е д с т в и е 6. Если для некоторого $k$ выполняются однородные условия согласования, то может существовать такой номер $n<k, n \in \mathbb{N}$, что для всех $0 \leq m \leq n, m \in \mathbb{N}$, выполняются неоднородные условия согласования (10).

Таким образом, из леммы 2 и следствий 3-6 получена следующая теорема.

Т е о р е м а 2. Пусть $\lambda \in C^{1}(\bar{Q}), q^{(j)} \in C^{2}([0 ;+\infty)), K_{j} \in C^{2}(\bar{Q}), j \in\{0, l\}, \quad \varphi \in C^{2}([0 ; l])$, $\psi \in C^{1}([0 ; l])$. Решение задачи (4)-(6), определяемое (9) и удовлетворяющее условиям сопряжения (11), (12), является единственным классическим в области $\tilde{Q}$ тогда и только тогда, когда выполнены неоднородные условия согласования

$$
\begin{gathered}
q^{(0)}(0)-\varphi(0)+\int_{0}^{l} K_{0}(0, s) \varphi(s) d s=\sigma_{0}^{(0)}, q^{(l)}(0)-\varphi(l)+\int_{0}^{l} K_{l}(0, s) \varphi(s) d s=\delta_{0}^{(0)}, \\
-\frac{1}{a} d q^{(0)}(0)+\frac{1}{a} \psi(0)-\frac{1}{a} \int_{0}^{l}\left(\partial_{t} K_{0}(0, s) \varphi(s)+\psi(s) K_{0}(0, s)\right) d s=\sigma_{1}^{(0)}, \\
\frac{1}{a} d q^{(l)}(0)-\frac{1}{a} \psi(l)-\frac{1}{a} \int_{0}^{l}\left(\partial_{t} K_{l}(0, s) \varphi(s)+\psi(s) K_{l}(0, s)\right) d s=\delta_{1}^{(0)}, \\
\frac{1}{a^{2}} d^{2} q^{(0)}(0)-d^{2} \varphi(0)-\frac{1}{a^{2}} \varphi(0) \lambda(0,0)+ \\
+\frac{1}{a^{2}} \int_{0}^{l}\left(\partial_{t}^{2} K_{0}(0, s) \varphi(s)+2 \partial_{t} K_{0}(0, s) \psi(s)+K_{0}(0, s)\left(a^{2} d^{2} \varphi(s)+\varphi(s) \lambda(0, s)\right)\right) d s=\sigma_{2}^{(0)}, \\
\frac{1}{a^{2}} d^{2} q^{(l)}(0)-d^{2} \varphi(l)-\frac{1}{a^{2}} \varphi(l) \lambda(0, l)+ \\
+\frac{1}{a^{2}} \int_{0}^{l}\left(\partial_{t}^{2} K_{l}(0, s) \varphi(s)+2 \partial_{t} K_{l}(0, s) \psi(s)+K_{l}(0, s)\left(a^{2} d^{2} \varphi(s)+\varphi(s) \lambda(0, s)\right)\right) d s=\delta_{2}^{(0)} .
\end{gathered}
$$

Неоднородное уравнение с неоднородными условиями согласования. Рассмотрим теперь неоднородное уравнение (1). В силу линейности, решение $v(t, x)$ данного уравнения можно представить как сумму решения $u(t, x)$ однородного уравнения и некоторого частного решения 
$w(t, x)$ неоднородного уравнения, т. е. $v(t, x)=u(t, x)+w(t, x)$. В качестве задачи для поиска некоторого частного решения $w(t, x)$ рассмотрим задачу $(7),(8)$.

Как было показано в [5], решение $w(t, x)$ задачи $(7),(8)$ существует в классе $C^{2}(\bar{Q})$ при выполнении условий $\lambda, f \in C^{1}(\bar{Q})$. Таким образом, для задачи (1)-(3) справедлива следующая теорема.

Т е о р е м а 3. Пусть $\lambda, f \in C^{1}(\bar{Q}), q^{(j)} \in C^{2}([0 ;+\infty)), K_{j} \in C^{2}(\bar{Q}), j \in\{0, l\}, \varphi \in C^{2}([0 ; l])$, $\psi \in C^{1}([0 ; l])$. Решение задачи (1)-(3), удовлетворяющее условиям сопряжения (11), (12), является единственныл классическим в области $\tilde{Q}$ тогда и только тогда, когда выполнены неоднородные условия согласования

$$
\begin{gathered}
\widetilde{q^{(0)}}(0)-\varphi(0)+\int_{0}^{l} K_{0}(0, s) \varphi(s) d s=\sigma_{0}^{(0)}, \widetilde{q^{(l)}}(0)-\varphi(l)+\int_{0}^{l} K_{l}(0, s) \varphi(s) d s=\delta_{0}^{(0)}, \\
-\frac{1}{a} \widetilde{d q^{(0)}}(0)+\frac{1}{a} \psi(0)-\frac{1}{a} \int_{0}^{l}\left(\partial_{t} K_{0}(0, s) \varphi(s)+\psi(s) K_{0}(0, s)\right) d s=\sigma_{1}^{(0)}, \\
\frac{1}{a} \widetilde{q^{(l)}}(0)-\frac{1}{a} \psi(l)-\frac{1}{a} \int_{0}^{l}\left(\partial_{t} K_{l}(0, s) \varphi(s)+\psi(s) K_{l}(0, s)\right) d s=\delta_{1}^{(0)}, \\
+\frac{1}{a^{2}} \int_{0}^{l}\left(\partial_{t}^{2} K_{0}(0, s) \varphi(s)+2 \partial_{t} K_{0}^{(0)}(0)-d^{2} \varphi(0)-\frac{1}{a^{2}}(\varphi(0) \lambda(0,0)+f(0,0))+\right. \\
\frac{1}{a^{2}} d^{2} \widetilde{q^{(l)}}(0)-d^{2} \varphi(l)-\frac{1}{a^{2}}(\varphi(l) \lambda(0, l)+f(0, l))+ \\
+\frac{1}{a^{2}} \int_{0}^{l}\left(\partial_{t}^{2} K_{l}(0, s) \varphi(s)+2 \partial_{t} K_{l}(0, s) \psi(s)+K_{l}(0, s)\left(a^{2} d^{2} \varphi(s)+\varphi(s) \lambda(0, s)+f(0, s)\right)\right) d s=\delta_{2}^{(0)} .
\end{gathered}
$$

Заключение. В данном сообщении рассмотрена смешанная задача для уравнения типа Клейна-Гордона-Фока с интегральными условиями в случае, когда выполняются неоднородные условия согласования. Поставлена эквивалентная задача сопряжения, результаты которой можно применять при численном моделировании.

Отдельно стоит отметить, что в отличие от первой смешанной задачи [7], в которой разрывы $\delta_{i}^{(k)}, \sigma_{i}^{(k)}, i=\overline{0,2}, k=0,1, \ldots$, сохраняются, в задаче с интегральными условиями разрывы на характеристиках могут быть сглажены, начиная с некоторой области $Q^{(k)}$, при выполнении условий следствия 5.

\section{Список использованных источников}

1. Корзюк, В. И. Первая смешанная задача для уравнения Клейна-Гордона-Фока в полуполосе / В. И. Корзюк, И. И. Столярчук // Дифференц. уравнения. - 2014. - Т. 50, № 8. - С. 1105-1117. https://doi.org/10.1134/s0374064114080081

2. Корзюк, В. И. Классическое решение первой смешанной задачи для уравнения Клейна-Гордона-Фока в криволинейной полуполосе / В. И. Корзюк, И. И. Столярчук // Докл. Нац. акад. наук Беларуси. - 2014. - Т. 58, № 3. - С. 9-15.

3. Корзюк, В. И. Классическое решение первой смешанной задачи для гиперболического уравнения второго порядка в криволинейной полуполосе с переменными коэффициентами / В. И. Корзюк, И. И. Столярчук // Дифференц. уравнения. - 2017. - Т. 53, № 1. - С. 77-88. https://doi.org/10.1134/s0374064117010071

4. Корзюк, В. И. Классическое решение смешанной задачи для уравнения Клейна-Гордона-Фока с нелокальными условиями / В. И. Корзюк, И. И. Столярчук // Докл. Нац. акад. наук Беларуси. - 2017. - Т. 61, № 6. - С. $20-27$.

5. Корзюк, В. И. Классическое решение смешанной задачи для уравнения Клейна-Гордона-Фока с нелокальными условиями / В. И. Корзюк, И. И. Столярчук // Тр. Ин-та математики. - 2018. - Т. 26, № 1. - С. 54-70.

6. Корзюк, В. И. Классические решения задач для гиперболических уравнений: Курс лекций в 10 ч. / В. И. Корзюк, И. С. Козловская. - Минск, 2017. - Ч. 2. - 52 с.

7. Корзюк, В. И. Классическое решение первой смешанной задачи для уравнения типа Клейна-Гордона-Фока с неоднородными условиями согласования / В. И. Корзюк, И. И. Столярчук // Докл. Нац. акад. наук Беларуси. - 2019. Т. 63, № 1. - C. 7-13. https://doi.org/10.29235/1561-8323-2019-63-1-7-13 


\section{References}

1. Korzyuk V. I., Stolyarchuk I. I. Classical solution of the first mixed problem for the Klein-Gordon-Fock equation in a half-strip. Differential Equations, 2014, vol. 50, no. 8, pp. 1098-1111. https://doi.org/10.1134/s0012266114080084

2. Korzyuk V. I., Stolyarchuk I. I. Classical solution to the first mixed problem for Klein-Gordon-Fock equation in the curvilinear half-strip. Doklady Natsional'noi akademii nauk Belarusi = Doklady of the National Academy of Sciences of Belarus, 2014, vol. 58, no. 3, pp. 9-15 (in Russian).

3. Korzyuk V. I., Stolyarchuk I. I. Classical solution of the first mixed problem for second-order hyperbolic equation in curvilinear half-strip with variable coefficients. Differential Equations, 2017, vol. 53, no. 1, pp. 74-85. https://doi.org/ $10.1134 / \mathrm{s} 0012266117010074$

4. Korzyuk V. I., Stolyarchuk I. I. Classical solution to the mixed problem for the Klein-Gordon-Fock equation with the unlocal conditions. Doklady Natsional'noi akademii nauk Belarusi = Doklady of the National Academy of Sciences of Belarus, 2017, vol. 61, no. 6, pp. 20-27 (in Russian).

5. Korzyuk V. I., Stolyarchuk I. I. Classical solution to the mixed problem for the Klein-Gordon-Fock equation with the unlocal conditions. Trudy Instituta matematiki = Proceedings of the Institute of Mathematics, 2018. vol. 26, no. 1, pp. 54-70 (in Russian).

6. Korzyuk V. I., Kozlovskaya I. S. Classical problem solutions for hyperbolic equations: A course of lectures in 10 parts. Minsk, 2017, part 2. 52 p. (in Russian).

7. Korzyuk V. I., Stolyarchuk I. I. Classical solution of the first mixed problem for the Klein-Gordon-Fock equation with inhomogeneous matching conditions. Doklady Natsional'noi akademii nauk Belarusi = Doklady of the National Academy of Sciences of Belarus, 2019, vol. 63, no. 1, pp. 7-13 (in Russian). https://doi.org/10.29235/1561-8323-2019-63-1-7-13

\section{Информация об авторах}

Корзюк Виктор Иванович - академик, д-р физ.-мат. наук, профессор. Институт математики НАН Беларуси (ул. Сурганова, 11, 220072, Минск, Республика Беларусь). E-mail:korzyuk@bsu.by.

Столярчук Иван Игоревич - магистр физ.-мат. наук, аспирант. Белорусский государственный университет (пр. Независимости, 4, 220030, Минск, Республика Беларусь). E-mail: ivan.telkontar@gmail.com.

\section{Information about the authors}

Korzyuk Viktor Ivanovich - Academician, D. Sc. (Physics and Mathematics), Professor. Institute of Mathematics of the National Academy of Sciences of Belarus (11, Surganov Str., 220072, Minsk, Republic of Belarus). E-mail: korzyuk@bsu.by.

Stolyarchuk Ivan Igorevich - Master of Physics and Mathematics, Postgraduate student. Belarusian State University (4, Nezavisimosti Ave., 220030, Minsk, Republic of Belarus). E-mail: ivan.telkontar@gmail.com. 